failure ; the intensive treatment was abandoned and the patients were now given the routine course. Women as well as men had been treated equally successfully with no distinction in dosage either in respect of body-weight or of sex. Three pregnant women had been treated with a 30-day course ; 2 had given birth to healthy babies and the third was due to be confined soon.

Maj. Walker said that she agreed entirely with Sqn.-ldr. Morton in her observations with regard to the intensive courses for women. She had been giving 3 injections weekly of 0.06 gramme of mapharside and, apart from 2 cases of erythema of the ninth day and a primary rise of temperature, there had been no untoward reactions. She too had noticed the gloomy irritability of some of the girls and agreed that the medical officer was largely dependent upon the observations of the nursing staff, which needed to be recorded accurately.

Sqn.-Idr. Moynihan said that he had treated primary and secondary cases of syphilis with 3 injections per week of mapharside. The patients were kept in bed under observation throughout treatment. Every case without exception progressed smoothly and the outcome was successful ; he soon ceased keeping them in bed and allowed them to get up. Eventually all cases were treated as out-patients and attended 3 times each week. He had had no experience of the toxic dermal reactions described by Maj. Marshall. Blood counts and serological tests were made weekly ; the urine was examined and the patients were instructed to take their temperature the day after the injections; but nothing untoward had happened. The blood counts were usually higher at the end of treatment than at the beginning. The weight of the patients increased, and this was not due to feeding in the hospital because they were fed by their respective units. The ambulant method of treatment with mapharside had very great advantages if the cases were properly selected and supervised.

Dr. Heywood said that he had had very little difficulty in persuading patients to come into hospital for the intensive treatment of syphilis, even though he was dealing with a very large working population in an industrial town. The question of being "off work " was always covered by a medical certificate and, in cases in which the social stigma of a certificate from the venereologist might be objected to, the patient could always be sent to his own medical practitioner and be quite certain that the truth would never be revealed. One or two female patients had objected to entering hospital. Young people in the age group 16-20 years were difficult to persuade to undergo any form of treatment ; in a really bad case two health visitors were sent to the home in a car to bring the patient into hospital.

Surg.-Lieut. Cdr. F. G. Maitland, in reply, recalled the fact that in his scheme a different dosage was given to each of the three separate groups of primary syphilis patients undergoing intensive treatment. He had recognized two types of jaundice, and he thanked Prof. Beattie and Maj. Marshall for their collaboration and help in the treatment of this complication.

\title{
Penicillin therapy for venereal diseases
}

In a general survey of penicillin therapy in the armed Forces in Canada, Farquharson, Greey and Townsend report on the treatment of gonococcal urethritis in over 1,000 males and in 110 females. For male patients the dosage was $90,000-120,000$ units, usually given over a period of 15 hours, but as little as 50,000 units sufficed in some cases if not more than 10 hours intervened between the first and last doses. For women the total dosage was usually 200,000 units, given intramuscularly in 20 injections at 3-hourly intervals. In only 67 female cases was there bacteriological confirmation of the clinical diagnosis at the time of treatment. There were 5 cases in which treatment was undoubtedly unsuccessful. Penicillin therapy for syphilis was used principally for patients who were believed to be intolerant of arsenic. In a few sero-positive cases of latent syphilis (which were treated with 2,000,000 units, given in 100 doses of 20,000 units each at 3-hourly intervals) the titre gradually decreased over a period of 6 months in all but 2 cases, and in one case the reaction became negative.-Canadian Medical Association Journal, July 1945.

\section{Ambulatory penicillin treatment of sulphonamide-resistant gonorrhoea}

Fidler, of the County Venereal Disease Clinic, Hopkinsville (Ky), has treated with penicillin 54 patients with sulphonamide-resistant gonorrhoea who were attending the out-patient clinic for venereal diseases. They were given 100,000 units of penicillin in 3 doses (each of 33,333 units) at 2-hourly intervals.

The patients were of both sexes ( 16 male, 38 female) and their ages ranged from under 10 to over 50 years. An adequate follow-up was possible in only 42 of the cases, but of these 95 per cent "satisfied the established criterion of cure ": 3 consecutive negative cultures within not less than 21 days after the first treatment.-Journal of Venereal Disease Information, July 1945. 